\title{
Are objective 'findings' the same as subjective 'severity'? A study of the relationship between computed tomography findings and subjective severity in preoperative CRSwNP patients
}

\author{
HANYU LU ${ }^{1}$, ZHUOFU LIU ${ }^{1}$, LI HU ${ }^{1}$, XIAN FENG ${ }^{2}$, LI HU ${ }^{1}$, YURONG GU ${ }^{1}$, \\ HOUYONG LI ${ }^{1}$, HUABIN LI $^{1}$ and DEHUI WANG ${ }^{1}$ \\ Departments of ${ }^{1}$ Otorhinolaryngology and ${ }^{2}$ Clinical Laboratory, Eye, Ear, \\ Nose and Throat Hospital of Fudan University, Shanghai 200031, P.R. China
}

Received October 14, 2018; Accepted January 22, 2020

DOI: $10.3892 / \mathrm{etm} .2020 .9032$

\begin{abstract}
In pre-surgical patients with chronic rhinosinusitis with nasal polyps (CRSwNPs), positive findings on computed tomography (CT) scans and an exacerbation of symptoms are two possible factors that indicate surgery. However, the relationship between these factors remains unclear. Confirmed CRSwNP patients ready for sinus surgery were consecutively enrolled. The Sino-Nasal Outcome Test 22 (SNOT-22) and visual analog scale (VAS) scoring were completed by these patients, and scores were subjected to factor analysis using a principal component analysis (PCA) to evaluate subjective symptom components related to CRS. Patient CT scores, measured by the Lund-Mackay score (LMS), together with their demographics, medical treatment, and atopy status, were collected and analyzed. A total of 288 patients who met the criteria for CRSwNP and underwent CT scanning prior to surgery in the Eye, Ear, Nose, and Throat Hospital of Fudan University were enrolled. Five components were identified from the PCA of the SNOT-22 results and VAS scores related to subjective symptoms. More severe 'nasal' symptoms ( $\mathrm{P}=0.03 ; 95 \% \mathrm{CI}, 0.092-1.824)$, loss of smell and taste $(\mathrm{P}<0.001 ; 95 \% \mathrm{CI}, 0.961-1.767)$ and lower facial pain ( $\mathrm{P}=0.001 ; 95 \%$ CI 1.811 to -0.475$)$, derived from the SNOT-22, were significantly associated with total CT score. For the VAS PCA components, less severe 'ocular' symptoms $(\mathrm{P}=0.004$; 95\% CI -1.852 to -0.352), a reduced 'pain' component $(\mathrm{P}<0.001$; 95\% CI -2.133 to -0.715), a higher 'nasal' symptom component $(\mathrm{P}<0.001 ; 95 \% \mathrm{CI} 0.752-2.257)$ and, again, loss of smell and taste $(\mathrm{P}<0.001 ; 95 \%$ CI $0.437-0.811)$ showed an association with total CT score. 'Allergy-associated symptoms' in both
\end{abstract}

Correspondence to: Dr Dehui Wang, Department of Otorhinolaryngology, Eye, Ear, Nose and Throat Hospital of Fudan University, 83 Fenyang Road, Shanghai 200031, P.R. China

E-mail: wangdehuient@sina.com.cn

Key words: chronic rhinosinusitis, health-related quality of life, factor analysis, Lund-Mackay score the SNOT-22 and VAS scores and 'loss of smell or taste' and 'hyposmia' were significantly more severe in patients with ethmoid sinus/maxillary sinus (E/M) ratios of $>2.59$ compared with patients with $\mathrm{E} / \mathrm{M}$ ratios $<2.59$. Loss of smell and taste alone was correlated with the LMS in patients with highly positive CT scores. The present study demonstrated associations between several subjective symptoms and objective severity in preoperative CRSwNP patients. Nasal symptoms, including olfaction loss, were correlated positively with CT scores, while ocular- and pain-related symptoms showed an opposite pattern. However, this relationship was not confirmed in patients with highly positive CT scores. A subset analysis confirmed, in terms of symptoms, the value of the E/M ratio for indicating eosinophilic chronic rhinosinusitis.

\section{Introduction}

The definition of chronic rhinosinusitis (CRS) is proposed in two guidelines as including the presence of nasal obstructions, nasal discharge, loss of smell and facial pain; however, the relationship with subjective symptoms pertinent to CRS is intricate $(1,2)$. The major complaints of patients with CRS, indeed tend to be sinus-specific, including nasal congestion and discharge, facial pain/pressure and/or olfactory disturbances. In addition, some of the less common symptoms, such as fatigue and headache, which are not relevant to sinusitis pathology but rather to a host of other chronic conditions, have also been shown in several studies to have an effect on disease severity (3-5) In previous studies, psychiatric distress, including workplace disturbances, anxiety and depression, have been reported to be associated with increased symptom burden in CRS patients $(6,7)$ Accordingly, several validated patient-reported measures of outcomes have been utilized since the 1990s to evaluate health-related quality of life. Among them, the Sino-Nasal Outcome Tests (SNOTs), including versions such as the SNOT-16, SNOT-2, and Sino-Nasal Assessment Questionnaire 11 (SNAQ-11), which is a modification of the 31-question Rhinosinusitis Outcome Measure (RSOM-31), has mostly been applied in the past decade (8-10).

Endoscopic signs of nasal polyps or edema in the middle meatus have invariably been considered objective evidence of 
rhinosinusitis. A well-performed evaluation by an experienced rhinologist using endoscopy can be pivotal in measuring mucosal inflammation during the primary diagnosis of the condition (11). Computed tomography (CT) scanning, however, due to its better ability to display the paranasal sinus and the soft tissue, is used frequently following medical treatment failure. Kennedy (12) suggested in 1992 that the extent of a disease evaluated by $\mathrm{CT}$ was the only potential factor affecting surgical outcomes. In addition, CT scans can provide endoscopic visualization of sinus tissue abnormalities and allow more rigorous assessment when referenced to a grading system (13). This is in addition to the practical difficulties of obtaining a clear endoscopic picture for primary unit physicians.

Nasal polyps appear in almost $20 \%$ of CRS patients and may increase the severity of mucosal inflammation (1). It has been reported that positive radiological findings are more common in patients with CRS with nasal polyps compared with those without (14). In addition, complaint profiles between patients with the two phenotypes of CRS are quite different. In CRSwNP patients who fail to see improvements following adequate medical treatment, surgical interventions may generally be the next option. When evaluating medical treatments and making the decision for surgery, radiological findings and self-reported symptoms are the most frequently used assessments (15). Any positive radiological findings or deterioration in self-reported symptoms may contribute to a choice for surgery. Therefore, it is of great significance to determine the severity of symptoms and the objective nasal condition prior to surgery. However, the correlation between these two signs remains controversial. Several previous studies using cumulative scores from a symptom questionnaire failed to confirm the relationship of self-reported symptoms with CT scores $(10,11,15)$. This may be due to the diversity in the spectrum of symptoms, as some of the symptoms cannot be explained by the severity of sinus inflammation and thus do not correlate with radiological findings. Therefore, to determine which symptoms actually relate to CT scores, these questionnaires must be subcategorized. Kenny et al (16) selected 7 commonplace symptoms and demonstrated that 5 of them were correlated with CT scores in patients with CRS or acute rhinosinusitis. Bhattacharyya (17) examined 115 adult patients with CRSwNP using the Rhinosinusitis Symptom Inventory (RIS), which has 4 domains for symptom evaluation, and found that none of the domains correlated with Lund scores. Notable among these studies was the use of factor analysis to make the research clinically meaningful. Browne et al (8) first proposed the use of subscales derived by factor analysis. Recent studies then compared the constructs of the SNOTs with Lund scores in patients both with and without nasal polyps and detected a relation only for the nasal symptom subset $(18,19)$. Sedaghat et al $(19)$ also found that many confounding factors such as sex and disease history may influence subjective symptoms.

The present study evaluated the associations between disease severity, measured by CT scores, and symptoms, while controlling for several confounding demographic factors, among preoperative CRSwNP patients. In addition, in order to avoid the flaws in the Lund-Mackay score (LMS) caused by the large range of the scoring interval (0-24) and in consideration of the possibility of a relationship between the symptoms of patients with subtle or less positive CT scores and the LMS, a subgroup analysis of patients with 'very positive' CT scores was performed. It was hypothesized that the constructs of the SNOT-22 that were associated with symptoms may be associated with radiological findings when adjusted for demographic covariances and that these differences may exist in patients with a more severe condition as shown by CT scores.

\section{Materials and methods}

Participants and setting. The present study was conducted at the Department of Otolaryngology, Head and Neck Surgery at the Affiliated Eye, Ear, Nose and Throat Hospital of Fudan University. A total of 288 adult CRSwNP patients ( $>18$ years) referred for endoscopic sinus surgery were consecutively recruited between September 2015 and February 2017. Diagnoses of CRSwNP were in accordance with the criteria defined by the EPOS 2012 position paper as the presence of two or more specific symptoms together with endoscopic signs of nasal polyps (20). All the patients were admitted for sinus surgery following unsuccessful previous medical treatment for 3 continuous months. All the participants were followed up for at least 3 months post-surgery. Exclusion criteria included acute exacerbation of a severe disease or the existence of conditions that may affect the mucosa of the sinus or nasal cavities, such as trauma, tumors or choanal polyp before surgery. Participants were also excluded if they had not undergone a CT scan within 1 month prior to surgery. Patient details are given in Table I.

Ethical considerations. The present study was approved by the Ethics Committee of the Affiliated Eye, Ear, Nose and Throat Hospital of Fudan University (Shanghai, China) and was conducted in accordance with the Declaration of Helsinki. Written consent was obtained from patients following they had been given written and oral information about the study and the potential side effects.

\section{Indicators and measurement tools}

Demographic information. Demographic information, including sex, age, medication history, duration of symptoms, history of previous surgeries, concurrent asthma, cigarette smoking and allergies to relevant airborne allergens were collected (Table I). The atopic status of the patients was evaluated by a skin prick test or an ImmunoCAP test (Phadia, Thermo Fisher Scientific, Inc.) to detect IgE antibodies against various common inhalant allergens.

CT scanning. CT scanning was performed using 1-mm axial images, which were reconstructed offline and reformatted to 3-mm coronal images for analysis (Toshiba Aquilion, Toshiba Medical Systems). All CT scans were scored following the Lund-Mackay system by an independent radiologist (21). The total score was the total LMS, with a maximum of 16 points. A total LMS of 0 was classified as negative; a unilateral LMS of 10 to 12 or a bilateral LMS of 12 to 24 was classified as 'very positive'. The ethmoid sinus/maxillary sinus (E/M) ratio was defined as the ratio of the CT-derive ethmoid sinus and maxillary sinus scores (22). 
Table I. Demographics of subjects.

\begin{tabular}{lc}
\hline Items & CRSwNP patients $(\mathrm{n}=288)$ \\
\hline Age (years) & $46.5 \pm 14.5(18-79)$ \\
Sex & 64.2 \\
Male (\%) & 35.8 \\
Female (\%) & 28.8 \\
Smoking (\%) & 25.3 \\
Atopy (\%) & 9.0 \\
Asthma history (\%) & 23.6 \\
Previous surgery (\%)
\end{tabular}

CRSwNP, chronic rhinosinusitis with nasal polyps.

Subjective symptoms. Patients' subjective symptoms were evaluated using two questionnaires around the time of the CT scan. A visual analog scale (VAS) was used to assess major and minor symptoms according to several guidelines. The symptoms assessed by this questionnaire included the following: Nasal obstructions, purulent nasal discharge, watery rhinorrhea, nasal itching, sneezing, facial pain, headache, itchy eyes, watery eyes, red eyes, eye pain, suffocation feeling, gasping for breath and oppressive feelings. Patients indicated their subjective symptoms on a straight line from 0 to $10 \mathrm{~cm}$ to describe the intensity of the symptoms. The SNOT is a patient-reported measure of outcomes in nasal and sino-nasal disorders, which has been validated and is commonly used in health-related quality of life assessments to describe patients' burdens and clinical effectiveness (23). This questionnaire consists of 22 items covering physical and functional problems together with emotional disorders.

Data analysis. A regression model was selected to fit previously considered confounders of the symptoms related to CRS with a lower likelihood of an association with CT scores. Redundant non-significant independent variables (multivariate P-value $>0.100$ ) were removed from the multivariate model using backward elimination. All remaining variables were adjusted when evaluating the relationship between subjective symptoms and the LMS.

As the two questionnaires contained a total of 16 or 22 items, both were subjected to factor analysis using PCA with Kaiser normalizations and varimax rotations to identify the subset of principle symptom components for regression analysis. Components that met the criteria (initial eigenvalues $>1.0$ ) were identified and explained according to the related primary symptoms. Symptoms from the SNOT-22 and VAS with low communalities $(<0.5)$ were excluded from the factor analysis and analyzed separately. Statistical analyses were performed with SPSS version 19 (IBM Corp.).

\section{Results}

Characteristics of study subjects. In total, 369 patients who met the criteria for CRSwNP and were ready for sinus surgery were consecutively assessed for eligibility. A total of 81 participants were excluded based on the exclusion criteria, which left a total sample of 288 patients (185 male and 103 female) enrolled in this cross-sectional study. Clinical and demographic characteristics are listed in Table I. The mean age of the patients was $46.5 \pm 14.5$ years, $28.8 \%$ had a history of smoking, $25.3 \%$ had a concurrent atopy status, and $9 \%$ had a history of asthma. The average total LMS was 9.55 (standard deviation, 6.70), and the average SNOT-22 score was 23.18 (standard deviation, 21.02).

Factor analysis. Exploratory factor analysis using PCA was performed to identify the subscale factors that explained the variance in the CT scores. In the SNOT-22,3 items (loss of smell and taste, postnasal discharge and facial pain) and 2 items in the VAS (cough and hyposmia) were excluded from factor analysis in the first run as a result of the low communalities $(<0.5)$ that they conveyed. Following application of Kaiser normalization and varimax rotations, 5 unique constructs each were found within the SNOT-22 and VAS results. These constructs were then labeled in accordance with the topics they addressed, which were 'working disturbances', 'sleeping disorders', 'nasal symptoms', 'otology symptoms', and 'allergy-associated symptoms' within the SNOT-22 (Table II) and 'ocular symptoms', 'pectoral symptoms', 'allergy-associated symptoms', 'pain', and 'nasal symptoms' within the VAS (Table III). All factor scores were preserved for regression analysis.

Confounding factors. The patient characteristics measured included previous surgeries, history of asthma and other demographic characteristics, which are presented in Table III. The associations of age, sex, history of asthma and allergies, smoking status and previous endonasal surgery with CT scores were tested separately using backward elimination from the multivariate model. Smoking status, age and previous endonasal surgery were consecutively removed from the regression model on the basis of a multivariate P-value $>0.100$ in three runs. History of asthma, sex, and other characteristics were not included in the regression because they were not significant $(\mathrm{P}>0.1)$.

Associations between symptoms and CT score. The constructs of the SNOT-22/VAS and the items excluded from the factor analysis were assessed for associations with LMS, adjusted for 'sex' and 'history of asthma'. No significant association was identified between the total SNOT-22 score and the LMS, while a slightly positive association existed between the total VAS score and the LMS ( $\mathrm{P}=0.008)$. Several positive and negative correlations were found between constructs extracted from the SNOT-22 and VAS (Tables IV and V). The 'nasal symptoms' construct $(\mathrm{P}<0.001)$ and 'loss of smell and taste' construct $(\mathrm{P}<0.001)$ of the SNOT-22 as well as chest symptoms (PCA of the VAS; $\mathrm{P}=0.022$ ), nasal symptoms (PCA of the VAS; $\mathrm{P}<0.001)$ and 'hyposmia' $(\mathrm{P}<0.001)$ were positively correlated with the LMS. Meanwhile, otologic symptoms (PCA of the SNOT-22; $\mathrm{P}=0.008)$, 'facial pain' $(\mathrm{P}=0.018)$, ocular symptoms (PCA of the VAS; $\mathrm{P}=0.035$ ) and pain-related symptoms (PCA of the VAS; $\mathrm{P}=0.031$ ) were negatively associated with LMS. The analysis of a subset of patients with a 'very positive' LMS is also shown in Table IV. Items associated with olfaction were the only factors that had a strong relationship with the LMS in both subsets $(\mathrm{P}<0.001)$. A total of 3 factors, the 'sleeping disorder' construct in the SNOT-22, the 'otology symptoms' construct in the SNOT-22 and the 'ocular symptoms' construct 
Table II. Summary of factor analysis using PCA for the SNOT-22 questionnaire.

\begin{tabular}{|c|c|c|c|c|c|}
\hline Symptoms & $\begin{array}{l}\text { Working } \\
\text { disturbance }\end{array}$ & $\begin{array}{l}\text { Sleeping } \\
\text { disorder }\end{array}$ & $\begin{array}{c}\text { Nasal } \\
\text { symptoms }\end{array}$ & $\begin{array}{l}\text { Otology } \\
\text { symptoms }\end{array}$ & $\begin{array}{c}\text { Allergy } \\
\text { associated symptoms }\end{array}$ \\
\hline Need to blow nose & -0.021 & -0.119 & 0.424 & -0.003 & -0.074 \\
\hline Nasal obstruction & -0.052 & 0.006 & 0.297 & -0.085 & 0.046 \\
\hline Sneezing & -0.016 & -0.049 & -0.051 & -0.112 & 0.611 \\
\hline Running nose & -0.080 & -0.059 & 0.319 & -0.030 & 0.158 \\
\hline Cough & -0.059 & -0.073 & -0.081 & 0.076 & 0.558 \\
\hline Thick nasal discharge & -0.042 & -0.035 & 0.389 & 0.086 & -0.275 \\
\hline Ear fullness & -0.098 & -0.004 & 0.038 & 0.434 & -0.097 \\
\hline Dizziness & 0.031 & -0.132 & -0.032 & 0.354 & 0.033 \\
\hline Ear pain & -0.151 & 0.018 & -0.052 & 0.507 & -0.007 \\
\hline Difficulty falling asleep & -0.215 & 0.423 & -0.003 & 0.086 & -0.069 \\
\hline Wake up at night & -0.109 & 0.406 & -0.084 & -0.016 & -0.040 \\
\hline Lack of good night's sleep & -0.063 & 0.347 & -0.024 & -0.084 & -0.019 \\
\hline Wake up tired & 0.074 & 0.187 & -0.070 & -0.052 & -0.008 \\
\hline Fatigue & 0.121 & 0.131 & -0.033 & -0.120 & 0.005 \\
\hline Reduced productivity & 0.164 & 0.045 & 0.001 & -0.065 & -0.063 \\
\hline Reduced concentration & 0.242 & -0.061 & -0.033 & -0.097 & 0.016 \\
\hline Sad & 0.281 & -0.124 & -0.051 & -0.040 & -0.005 \\
\hline Frustrated/restless/irritated & 0.295 & -0.157 & -0.034 & -0.010 & -0.044 \\
\hline Embarrassed & 0.288 & -0.192 & 0.008 & -0.016 & -0.010 \\
\hline Variance explained & $21.617 \%$ & $14.565 \%$ & $13.724 \%$ & $11.517 \%$ & $7.558 \%$ \\
\hline
\end{tabular}

'Loss of smell and taste' (communality $=0.453$ ), 'post nasal discharge' (communality=0.465) and 'facial pain' (communality=0.454) were excluded from factor analysis and analyzed separately. Kaiser-Meyer-Olkin=0.906; Bartlett's sphericity, $\mathrm{P}<0.001\left[\chi^{2}=3212.3\right.$, degrees of freedom $(\mathrm{df})=171]$. The bold underlined figures for each construct represent the correlation of these variables that can be transferred to one independent component. PCA, principal component analysis; SNOT, Sino-Nasal Outcome Test.

Table III. Summary of factor analysis using PCA for VAS scores.

\begin{tabular}{lcccc}
\hline Symptoms & $\begin{array}{c}\text { Ocular } \\
\text { symptoms }\end{array}$ & $\begin{array}{c}\text { Asthma } \\
\text { associated symptoms }\end{array}$ & $\begin{array}{c}\text { Allergy } \\
\text { associated symptoms }\end{array}$ & $\begin{array}{c}\text { Nasal } \\
\text { symptoms }\end{array}$ \\
\hline Nasal obstruction & -0.047 & -0.027 & 0.083 & -0.075 \\
Thick nasal discharge & 0.029 & -0.103 & -0.117 & 0.020 \\
Running nose & -0.147 & 0.026 & 0.361 & -0.045 \\
Itchy nose & -0.006 & -0.097 & 0.391 & 0.053 \\
Sneezing & -0.003 & -0.079 & 0.430 & -0.042 \\
Facial pain/pressure & -0.050 & -0.135 & 0.030 & -0.067 \\
Headache & -0.128 & 0.020 & -0.060 & -0.085 \\
Itchy eyes & 0.329 & -0.112 & 0.084 & 0.0375 \\
Watery eyes & 0.325 & -0.072 & 0.009 & -0.045 \\
Swelling around eyes & 0.393 & -0.056 & -0.129 & -0.091 \\
Eye sore & 0.340 & -0.087 & -0.102 & -0.152 \\
Shortness of breath & -0.065 & 0.438 & -0.069 & -0.067 \\
Wheezing & -0.091 & 0.453 & -0.055 & 0.072 \\
Chest tightness & -0.110 & 0.418 & -0.016 & -0.007 \\
Variance explained & $20.114 \%$ & $17.242 \%$ & $16.311 \%$ & -0.064 \\
\hline
\end{tabular}

'Hyposmia' (communality=0.472) and 'cough' (communality=0.489) were excluded from factor analysis and analyzed separately. Kaiser-Meyer-Olkin=0.796; Bartlett's sphericity, $\mathrm{P}<0.001\left[\chi^{2}=1741.0\right.$, degrees of freedom $\left.(\mathrm{df})=91\right]$. The bold underlined figures for each construct represent the correlation of these variables that can be transferred to one independent component. PCA, principal component analysis; VAS, visual analog scale. 
Table IV. Relationship between the constructs of the SNOT-22 and LMS.

\begin{tabular}{|c|c|c|}
\hline Construct & $P(\alpha=0.05)$ & Estimate $(95 \% \mathrm{CI})$ \\
\hline \multicolumn{3}{|l|}{ SNOT-22 total score } \\
\hline Overall & 0.122 & $0.031(-0.008,0.070)$ \\
\hline In patients with very positive CT scores & 0.346 & $0.023(-0.026,0.072)$ \\
\hline \multicolumn{3}{|l|}{ 'Working disturbance' } \\
\hline Overall & 0.990 & $0.005(-0.837,0.848)$ \\
\hline In patients with very positive CT scores & 0.494 & $-0.298(-1.160,0.564)$ \\
\hline \multicolumn{3}{|l|}{ 'Sleeping disorder' } \\
\hline Overall & 0.267 & $-0.465(-1.288,0.358)$ \\
\hline In patients with very positive CT scores & 0.428 & $0.360(-0.540,1.259)$ \\
\hline \multicolumn{3}{|l|}{ 'Nasal symptoms’ } \\
\hline Overall & $<0.001$ & $1.594(0.805,2.384)$ \\
\hline In patients with very positive $\mathrm{CT}$ scores & 0.103 & $0.757(-0.155,1.669)$ \\
\hline \multicolumn{3}{|l|}{ ‘Otology symptoms’ } \\
\hline Overall & 0.008 & $-1.143(-1.980,0.306)$ \\
\hline In patients with very positive $\mathrm{CT}$ scores & 0.460 & $0.412(-0.693,1.516)$ \\
\hline \multicolumn{3}{|l|}{ 'Allergy associated symptoms' } \\
\hline Overall & 0.905 & $0.050(-0.779,0.879)$ \\
\hline In patients with very positive CT scores & 0.331 & $-0.441(-1.338,0.456)$ \\
\hline \multicolumn{3}{|l|}{ Loss of smell and taste } \\
\hline Overall & $<0.001$ & $1.276(0.904,1.648)$ \\
\hline In patients with very positive CT scores & $<0.001$ & $1.021(0.612,1.429)$ \\
\hline \multicolumn{3}{|l|}{ Postnasal discharge } \\
\hline Overall & 0.303 & $-0.278(-0.252,0.808)$ \\
\hline In patients with very positive CT scores & 0.983 & $0.006(-0.578,0.591)$ \\
\hline \multicolumn{3}{|l|}{ Facial pain } \\
\hline Overall & 0.018 & $-0.763(-1.395,0.131)$ \\
\hline In patients with very positive CT scores & 0.147 & $-0.606(-1.431,0.218)$ \\
\hline
\end{tabular}

SNOT, Sino-Nasal Outcome Test; LMS, Lund-Mackay score; CT, computed tomography.

in the VAS, showed a difference between the two subsets and the LMS. Though no significant difference was observed between the interaction of the 2 subsets with symptoms, this could indicate that in patients with different imaging findings, the causes of symptoms might also differ.

Subsets based on E/M Lund ratios. As shown in Figs. 1-4, when sorted by the E/M ratio, with a cutoff value of 2.59 , which was proposed in a previous study as an indication of CRS (eCRS) (22), 'allergy-associated symptoms' in both the SNOT-22 and the VAS, 'loss of smell or taste' and 'hyposmia' were significantly more severe in patients with an E/M ratio of $>2.59$ compared with those with a ratio $<2.59$. In addition, 'otologic symptoms' within the SNOT-22 showed less severity in patients with an E/M ratio $>2.59$.

\section{Discussion}

Judging the severity of CRS and assessing treatment options are predicated mainly upon clinical symptoms and radiographic findings. Previous studies of these two types of assessment have failed to confirm an established association $(10,11,15,17)$. It should be noted that several patients in previous studies met the criteria for CRS with no evidence of abnormalities on CT scans (24). By contrast, under the symptomatic definition of CRS, up to $20 \%$ of the 'normal' population also presented with incidental abnormalities in a previous study (21). Using different questionnaires, including the RSI, Chronic Sinusitis Survey, SNAQ and SNOTs, for symptom assessment, many earlier studies have indicated a lack of an association between symptoms and CT scores $(10,11,15-17,19,21,24)$. Among these questionnaires, the total score of the SNOT-20 or SNOT-22 (which is more commonly used) showed, with no exceptions, that there was no association with the LMS. Interestingly, Kenny et al (16) identified 5 out of 7 symptoms that were associated with the severity of CT scores, with the only exceptions being facial pain and headache. Although these questionnaires consist of many items, few studies have compared the relationship between specific symptoms and CT scores using these instruments. To address these problems, factor analyses have been applied to the results of these questionnaires. Several studies using factor analysis categorized 4 or 5 domains of the 
Table V. Relationship between constructs of VAS score and LMS.

\begin{tabular}{lcc}
\hline Construct & $\mathrm{P}(\alpha=0.05)$ & Estimate $(95 \% \mathrm{CI})$ \\
\hline 'Ocular symptoms' & & \\
Overall & 0.035 & $-0.912(-1.760,0.065)$ \\
In patients with very positive CT scores & 0.311 & $0.532(-0.507,1.571)$ \\
'Pectoral symptoms' & & \\
$\quad$ Overall & 0.022 & $0.955(0.141,1.769)$ \\
$\quad$ In patients with very positive CT scores & 0.446 & $0.309(-0.494,1.112)$ \\
'Allergy associated symptoms' & & \\
Overall & 0.982 & $0.009(-0.815,0.833)$ \\
In patients with very positive CT scores & 0.944 & $-0.031(-0.924,0.862)$ \\
'Pain' & & \\
Overall & 0.031 & $-0.933(-1.779,0.086)$ \\
In patients with very positive CT scores & 0.691 & $-0.230(-1.374,0.914)$ \\
'Nasal symptoms' & & \\
Overall & $<0.001$ & $2.294(1.513,3.075)$ \\
In patients with very positive CT scores & 0.068 & $0.796(-0.059,1.650)$ \\
Cough & & \\
Overall & 0.127 & $0.335(-0.095,0.765)$ \\
In patients with very positive CT scores & 0.534 & $0.135(-0.295,0.565)$ \\
Hyposmia & & \\
$\quad$ Overall & $<0.001$ & $0.690(0.512,0.867)$ \\
In patients with very positive CT scores & $<0.001$ & $0.531(0.314,0.748)$
\end{tabular}

VAS, visual analog scale; LMS, Lund-Mackay score; CT, computed tomography.

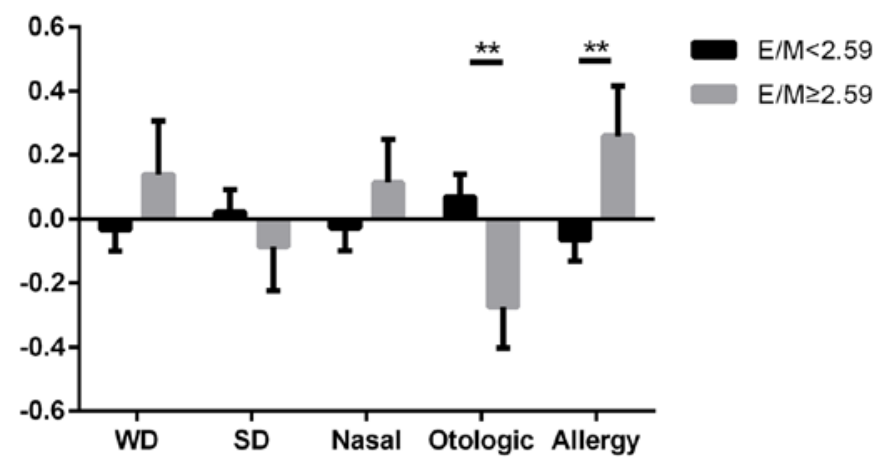

Figure 1. Components derived from SNOT-22 in patients subgroup with a CT scan score of $\mathrm{E} / \mathrm{M} \geq 2.59$ or $<2.59$. 'allergy-associated symptoms' were significantly more severe in patients with an $\mathrm{E} / \mathrm{M}$ ratio of $>2.59$ compared with those with a ratio $<2.59(\mathrm{P}=0.043)$. 'otologic symptoms' within the SNOT-22 showed less severity in patients with $\mathrm{E} / \mathrm{M}>2.59(\mathrm{P}=0.032){ }^{* *} \mathrm{P}<0.05$. SNOT, Sino-Nasal Outcome Test; CT, computed tomography; E/M, ethmoid sinus/maxillary sinus ratio; WD, working disturbance; SD, Sleeping disorder,

SNOT-20/22 and suggested that the questionnaire was actually evaluating more than one specific construct $(8,18,19,23,25)$. The present study also identified 5 constructs using PCA for factor analysis. These constructs broke down into 5 symptom-related domains: 'working disturbances', 'sleeping disorders', 'nasal symptoms', 'otology symptoms' and 'allergy-associated symptoms'. The symptoms in one specific domain may relate to a certain disease subtype or to health-related quality of life.

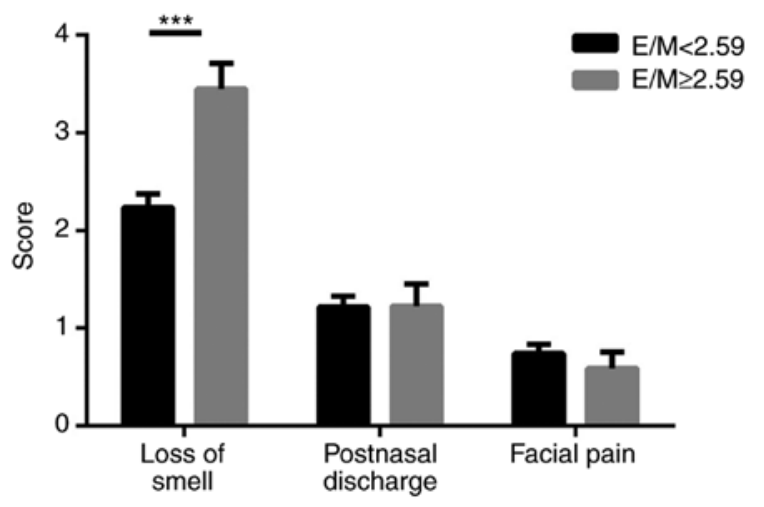

Figure 2. Excluded symptoms from SNOT-22 in patients subgroup with a CT scan score of $E / M \geq 2.59$ or $<2.59$. 'Loss of smell and taste' were significantly more severe in patients with an $\mathrm{E} / \mathrm{M}$ ratio of $>2.59$ compared with those with a ratio $<2.59(\mathrm{P}<0.001) .{ }^{* * *} \mathrm{P}<0.01$. SNOT, Sino-Nasal Outcome Test; $\mathrm{CT}$, computed tomography; E/M, ethmoid sinus/maxillary sinus ratio.

In two previous studies that used PCA to analyze the constructs of SNOTs that related to CT scores, only nasal symptoms were positively associated $(10,26)$. In the present study, following adjustment for the influential demographic factors of sex and history of asthma, several symptoms and symptom constructs that had a positive relationship with the LMS were found in this cohort. Olfaction and nasal symptoms, including running nose, nasal obstructions and thick nasal discharge specifically, showed quite strong correlations with CT scores 


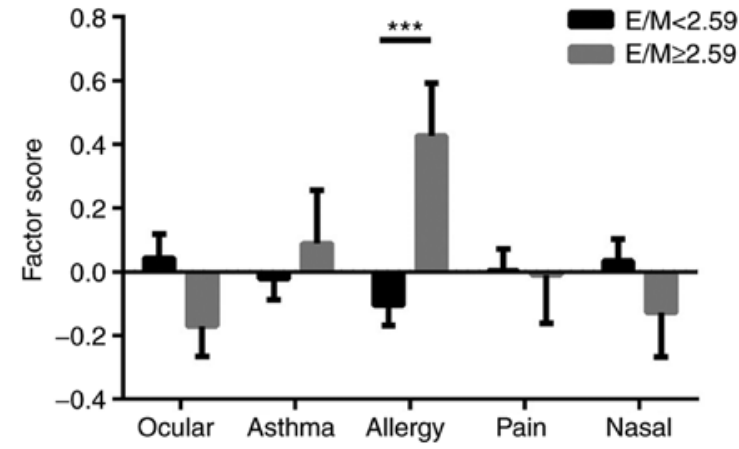

Figure 3. Components derived from VAS in patients subgroup with a CT scan score of $\mathrm{E} / \mathrm{M} \geq 2.59$ or $<2.59$. 'allergy-associated symptoms' were significantly more severe in patients with an $\mathrm{E} / \mathrm{M}$ ratio of $>2.59$ compared with those with a ratio $<2.59(\mathrm{P}=0.001) .{ }^{* * * *} \mathrm{P}<0.01$. VAS, visual analog scale; $\mathrm{CT}$, computed tomography; E/M, ethmoid sinus/maxillary sinus ratio.

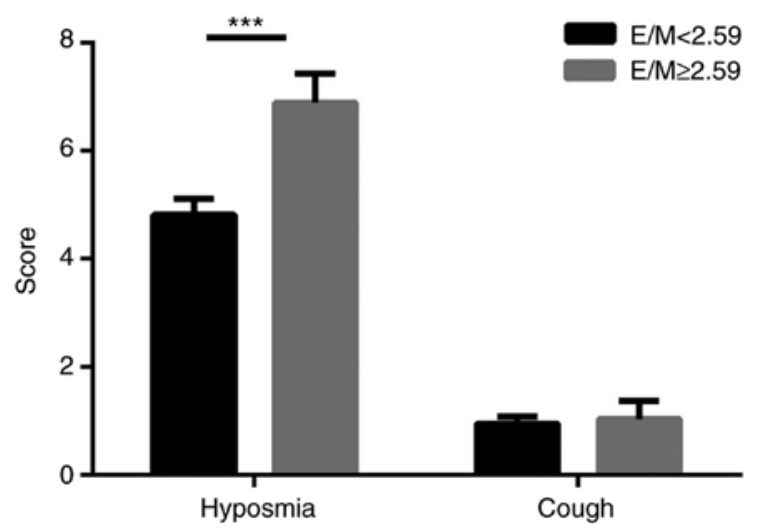

Figure 4. Excluded symptoms from VAS in patients subgroup with a CT scan score of $\mathrm{E} / \mathrm{M} \geq 2.59$ or $<2.59$. 'Hyposmia' were significantly more severe in patients with an $\mathrm{E} / \mathrm{M}$ ratio of $>2.59$ compared with those with a ratio $<2.59$ $(\mathrm{P}=0.001) .{ }^{* * *} \mathrm{P}<0.01$. VAS, visual analog scale; $\mathrm{CT}$, computed tomography; $\mathrm{E} / \mathrm{M}$, ethmoid sinus/maxillary sinus ratio.

in both the SNOT-22 and VAS questionnaires. Therefore, in patients with refractory nasal symptoms, radiological findings are more likely to be positive, which may make these patients more likely to consider a surgical option.

Meng et al (22) reported in a recent study that the E/M ratio based on CT scores may predict the phenotype of eCRS The cut-off value presented in that study was used to test for symptom differentiation between the two subsets. The results showed that olfaction- and allergy-associated symptoms were significantly more severe in patients with $\mathrm{E} / \mathrm{M}$ ratios $>2.59$, which coincided with the characteristics of eCRS reported in a previous study (27). This result confirmed the value of the $\mathrm{E} / \mathrm{M}$ ratio as a predictor for eCRS in terms of symptoms.

The negative correlation between 'pain' and CT scores in Gregurić et al (18) is also of interest. The present study also reported that pain-related symptoms were negatively associated with radiological findings. Similar results were reported previously in a long-term follow-up study that compared the relationship between facial pain and CT scores, where CRS patients with a primary symptom of facial pain tended to have negative $\mathrm{CT}$ scores (4). As the negative relationship did not exist in the subset of patients with CT scores of $>12$, the CT scores among 3 group of patients with L-M scores of 4-12, 13-24 and $<4$ were compared. Patients with relatively few findings on the CT scans and L-M scores of $<4$ tended to display higher facial pain symptom scores than the other 2 groups. The negative correlation was due to patients presenting with facial pain being more likely to have a negative CT scan appearance. As facial pain is among the major symptoms of CRS and motivates patients to seek medical attention, decisions should be made more carefully when clinicians encounter this kind of situation. In the cohort of the present study, some patients with a severe condition, as measured by CT scores, might still have had slight symptoms of CRS, and patients with extremely diseased unilateral lesions or extensive bilateral pathological changes were included in the analyzed groups. Patients with 'very positive' CT scores alone, however, can be considered to have undergone failed medical treatments. The present study investigated in particular the correlation between symptoms and radiological findings in these patients and found some subtle differences compared with the results of the overall cohort. In this subgroup of patients, only the loss of smell and taste remained associated with the LMS, which indicates a more complicated pattern of symptom severity in these patients. In patients with severe mucosal or sinus inflammation, the symptom of inflammation may be influenced by a threshold of sensation that different individuals feel quite differently.

The present study demonstrated the presence of a positive relationship between symptom subsets such as nasal symptoms and loss of smell, as well as a negative relationship between symptoms such as pain and ocular symptoms, with objective radiological findings in patients with pre-surgical CRSwNP. In addition, when sorted by the $\mathrm{E} / \mathrm{M}$ ratio, with a cutoff of 2.59, allergy-associated symptoms and olfactory symptoms showed significant differences between the 2 subgroups. In patients with very severe imaging findings, only a loss of smell remained correlated with the LMS.

\section{Acknowledgements}

The authors would like to thank Dr Wanpeng Li, Department of Otorhinolaryngology, Eye, Ear, Nose and Throat Hospital of Fudan University (Shanghai, China) for providing statistical advice in the present study.

\section{Funding}

This work was supported by the Natural Science Foundation of China (grant no. 81500781).

\section{Availability of data and materials}

All data generated or analyzed during this study are included in this published article or are available from the corresponding author on reasonable request.

\section{Authors' contributions}

Ha. $\mathrm{L}$ and $\mathrm{ZL}$ designed the study and wrote the manuscript. $\mathrm{LH}$ and XF were responsible for collecting and analyzing data. YG, Hou. L, Hua. L and DW were responsible for recruitment, carrying out the endoscopic examination. 


\section{Ethics approval and consent to participate}

The present study was approved by the Ethics Committee of the Affiliated Eye, Ear, Nose and Throat Hospital of Fudan University (approval no. 2016009; Shanghai, China) and was conducted in accordance with the Declaration of Helsinki. Written consent was obtained from patients following they had been given written and oral information about the study and the potential side effects.

\section{Patient consent for publication}

Not applicable.

\section{Competing interests}

The authors declare that they have no competing interests.

\section{References}

1. Fokkens WJ, Lund VJ, Mullol J, Bachert C, Alobid I, Baroody F, Cohen N, Cervin A, Douglas R, Gevaert P, et al: European position paper on rhinosinusitis and nasal polyps 2012. Rhinol Suppl 23: 3 p preceding table of contents, 1-298, 2012.

2. Benninger MS, Ferguson BJ, Hadley JA, Hamilos DL, Jacobs M, Kennedy DW, Lanza DC, Marple BF, Osguthorpe JD, Stankiewicz JA, et al: Adult chronic rhinosinusitis: Definitions, diagnosis, epidemiology, and pathophysiology. Otolaryngol Head Neck Surg 129 (Suppl 3): S1-S32, 2003.

3. Alt JA, Smith TL, Mace JC and Soler ZM: Sleep quality and disease severity in patients with chronic rhinosinusitis. Laryngoscope 123: 2364-2370, 2013.

4. Agius AM: Long-term follow-up of patients with facial pain in chronic rhinosinusitis-correlation with nasal endoscopy and CT. Rhinology 48: 65-70, 2010.

5. DeConde AS, Mace JC, Ashby S, Smith TL, Orlandi RR and Alt JA: Characterization of facial pain associated with chronic rhinosinusitis using validated pain evaluation instruments. Int Forum Allergy Rhinol 5: 682-690, 2015.

6. Davis GE, Yueh B, Walker E, Katon W, Koepsell TD and Weymuller EA: Psychiatric distress amplifies symptoms after surgery for chronic rhinosinusitis. Otolaryngol Head Neck Surg 132: 189-196, 2005.

7. Phillips KM, Hoehle LP, Bergmark RW, Campbell AP, Caradonna DS, Gray ST and Sedaghat AR: Association between nasal obstruction and risk of depression in chronic rhinosinusitis. Otolaryngol Head Neck Surg 157: 150-155, 2017.

8. Browne JP, Hopkins C, Slack R and Cano SJ: The sino-nasal outcome test (SNOT): Can we make it more clinically meaningful? Otolaryngol Head Neck Surg 136: 736-741, 2007.

9. Piccirillo JF, Edwards D, Haiduk A, Yonan C and Thawley SE: Psychometric and clinimetric validity of the 31-item rhinosinusitis outcome measure (RSOM-31). Am J Rhinol Volume 9: 297-306, 1995.

10. Basu S, Georgalas C, Kumar BN and Desai S: Correlation between symptoms and radiological findings in patients with chronic rhinosinusitis: An evaluation study using the sinonasal assessment questionnaire and lund-mackay grading system. Eur Arch Otorhinolaryngol 262: 751-754, 2005.
11. Ryan WR, Ramachandra $\mathrm{T}$ and Hwang $\mathrm{PH}$ : Correlations between symptoms, nasal endoscopy, and in-office computed tomography in post-surgical chronic rhinosinusitis patients. Laryngoscope 121: 674-678, 2011.

12. Kennedy DW: Prognostic factors, outcomes and staging in ethmoid sinus surgery. Laryngoscope 102 (Suppl 57): S1-S18, 1992.

13. Psaltis AJ, Li G, Vaezeafshar R, Cho KS and Hwang PH: Modification of the Lund-Kennedy endoscopic scoring system improves its reliability and correlation with patient-reported outcome measures. Laryngoscope 124: 2216-2223, 2014.

14. Deal RT and Kountakis SE: Significance of nasal polyps in chronic rhinosinusitis: Symptoms and surgical outcomes. Laryngoscope 114: 1932-1935, 2004.

15. Bradley DT and Kountakis SE: Correlation between computed tomography scores and symptomatic improvement after endoscopic sinus surgery. Laryngoscope 115: 466-469, 2005.

16. Kenny TJ, Duncavage J, Bracikowski J, Yildirim A, Murray JJ and Tanner SB: Prospective analysis of sinus symptoms and correlation with paranasal computed tomography scan. Otolaryngol Head Neck Surg 125: 40-43, 2001.

17. Bhattacharyya N: Relationship between mucosal inflammation, computed tomography, and symptomatology in chronic rhinosinusitis without polyposis. Ann Otol Rhinol Laryngol 117: 517-522, 2008.

18. Gregurić T, Trkulja V, Baudoin T, Grgic MV, Smigovec I and Kalogjera L: Association between computed tomography findings and clinical symptoms in chronic rhinosinusitis with and without nasal polyps. Eur Arch Otorhinolaryngol 274: 2165-2173, 2017.

19. Sedaghat AR, Gray ST, Caradonna SD and Caradonna DS: Clustering of chronic rhinosinusitis symptomatology reveals novel associations with objective clinical and demographic characteristics. Am J Rhinol Allergy 29: 100-105, 2015.

20. Fokkens WJ, Lund VJ and Mullol J: European position paper on rhinosinusitis and nasal polyps. Rhinology 45: 97-101, 2007.

21. Ashraf N and Bhattacharyya N: Determination of the 'incidental' Lund score for the staging of chronic rhinosinusitis. Otolaryngol Head Neck Surg 125: 483-486, 2001.

22. Meng $\mathrm{Y}$, Lou $\mathrm{H}$, Wang $\mathrm{C}$ and Zhang L: Predictive significance of computed tomography in eosinophilic chronic rhinosinusitis with nasal polyps. Int Forum Allergy Rhinol 6: 812-819, 2016.

23. DeConde AS, Mace JC, Bodner T, Hwang PH, Rudmik L, Soler ZM and Smith TL: SNOT-22 quality of life domains differentially predict treatment modality selection in chronic rhinosinusitis. Int Forum Allergy Rhinol 4: 972-979, 2014.

24. Ferguson BJ, Narita M, Yu VL, Wagener MM and Gwaltney JM Jr: Prospective observational study of chronic rhinosinusitis: Environmental triggers and antibiotic implications. Clin Infect Dis 54: 62-68, 2012.

25. DeConde AS, Bodner TE, Mace JC and Smith TL: Response shift in quality of life after endoscopic sinus surgery for chronic rhinosinusitis. JAMA Otolaryngol Head Neck Surg 140: 712-719, 2014.

26. Sedaghat AR and Bhattacharyya N: Chronic rhinosinusitis symptoms and computed tomography staging: Improved correlation by incorporating radiographic density. Int Forum Allergy Rhinol 2: 386-391, 2012.

27. Zuo K, Guo J, Chen F, Xu R, Xu G, Shi J and Li H: Clinical characteristics and surrogate markers of eosinophilic chronic rhinosinusitis in Southern China. Eur Arch otorhinolaryngol 271: 2461-2468, 2014

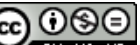

This work is licensed under a Creative Commons Attribution-NonCommercial-NoDerivatives 4.0 International (CC BY-NC-ND 4.0) License. 